\title{
An industrial approach to design compelling VR and AR experience
}

\author{
Simon Richir ${ }^{1}$, Philippe Fuchs ${ }^{2}$, Domitile Lourdeaux ${ }^{3}$, Cédric Buche $^{4}$, Ronan Querrec ${ }^{4}$ \\ ${ }^{1}$ Arts et Metiers ParisTech, LAMPA, 2 Bd du Ronceray, 49000 Angers - France \\ ${ }^{2}$ Mines ParisTech, VR\&RA Team ${ }^{3}$ UTC, Heudiasyc-UMR6599 \\ ${ }^{4}$ ENIB, LAB-STICC-UMR3192 \\ Contact author: simon.richir [a] ensam.eu
}

\begin{abstract}
The convergence of technologies currently observed in the field of VR, AR, robotics and consumer electronic reinforces the trend of new applications appearing every day. But when transferring knowledge acquired from research to businesses, research laboratories are often at a loss because of a lack of knowledge of the design and integration processes in creating an industrial scale product. In fact, the innovation approaches that take a good idea from the laboratory to a successful industrial product are often little known to researchers.

The objective of this paper is to present the results of the work of several research teams that have finalized a working method for researchers and manufacturers that allow them to design virtual or augmented reality systems and enable their users to enjoy "a compelling VR experience". That approach, called "the I2I method", present 11 phases from "Establishing technological and competitive intelligence and industrial property" to "Improvements" through the "Definition of the Behavioral Interface, Virtual Environment and Behavioral Software Assistance". As a result of the experience gained by various research teams, this design approach benefits from contributions from current VR \& AR research. Our objective is to validate and continuously move such multidisciplinary design team methods forward.
\end{abstract}

Keywords: Virtual reality, design process, human centered design, methodology, innovation, immersion, interaction

\section{THE I2I METHOD}

\subsection{Three levels of immersion and interaction}

The software and hardware production of a system integrating virtual reality cannot be designed without analyzing human behavior in a virtual world. From that point on, it becomes absolutely indispensible to work with cognitive science experts. Their role is to establish:

- The psychophysical characteristics of the user's senses and the motor responses;

- The conceptual and pragmatic differences between the schemas and metaphors employed in the system;

- The Behavioral Software Assistance needed to help the system user.

The limits of human behavior in relation to the solutions chosen by the system designers, in particular the human capacity to resolve any sensorimotor inconsistencies [4].

Our $\mathrm{I}^{2} \mathrm{I}$ approach is built around analyzing and modeling the subject's immersion and interaction in the virtual environment (Figure 1), developed at the Ecole des Mines ParisTech [3]. Modeling the subject's immersion and interaction should be examined on three levels:

- At a physical level, we talk about sensorimotor immersion and interaction ( $\left.\mathrm{I}^{2} \mathrm{sm}\right)$, as physically the computer is connected to the user through the user's senses and motor responses via the UPI (User Perceptual Interface) [1]. This level of immersion and interaction is quantifiable in relation to the characteristics of the senses and the motor responses being exploited. 
- The user must be mentally immersed in the virtual world. The "lower" level of sensorimotor immersion and interaction facing the user should be mentally invisible (transparent). In this case we talk about cognitive immersion and interaction $\left(\mathrm{I}^{2} \mathrm{c}\right)$. The cognitive processes (schemas, metaphors, substitutions) related to the interface are situated at this level.

- At a third level relating to the application of virtual reality, the objective is to endeavor to produce the immersion of the user for a given task (or a functionality). Here we talk about functional immersion and interaction $\left(\mathrm{I}^{2} \mathrm{f}\right)$.

This breakdown allows us to more effectively clarify the various issues encountered during the subject's (human being) immersion and interaction. They are closely linked and are not in conflict. The basis of our approach is this three level hierarchical model as well as a horizontal breakdown between the subject and the virtual world: in parallel to the person's various $\mathrm{I}^{2} \mathrm{sm}$ and $\mathrm{I}^{2} \mathrm{c}$ levels, we have two levels of software functioning for the virtual world. Symmetrically with the $\mathrm{I}^{2} \mathrm{sm}$, the computer has to manage the real time software part, allowing the physical production of the virtual world. This concerns the simulation based on physical laws (mechanical, optical, biomechanical, etc.) acting on the objects and animated beings.

Given the $\mathrm{I}^{2} \mathrm{c}$, the software part has to manage the behavior modeling for the virtual world. This software part has to provide a simulation of the behavior of the animated beings and the Behavioral Software Assistance (BSA) associated with the schemas used, in order to facilitate the person's cognitive $\mathrm{I}^{2} \mathrm{c}$.

In relation to the application and its objectives and at the $\mathrm{I}^{2} \mathrm{f}$ level, the following question must be asked: what must the user do? The subject's activities can always be broken down into a few basic behaviors we call "Virtual Behavioral Primitives" (VBP). Therefore at the $I^{2} f$ level the VBPs and their specificities must be properly established. Whatever the application, they can be grouped into four categories:

- observe the virtual world;

- move around in the virtual world;

- interact in the virtual world;

- communicate with others or with the application.

Our analysis of the modeling of the subject's immersion and interaction in the virtual environment is summed up by following schema:

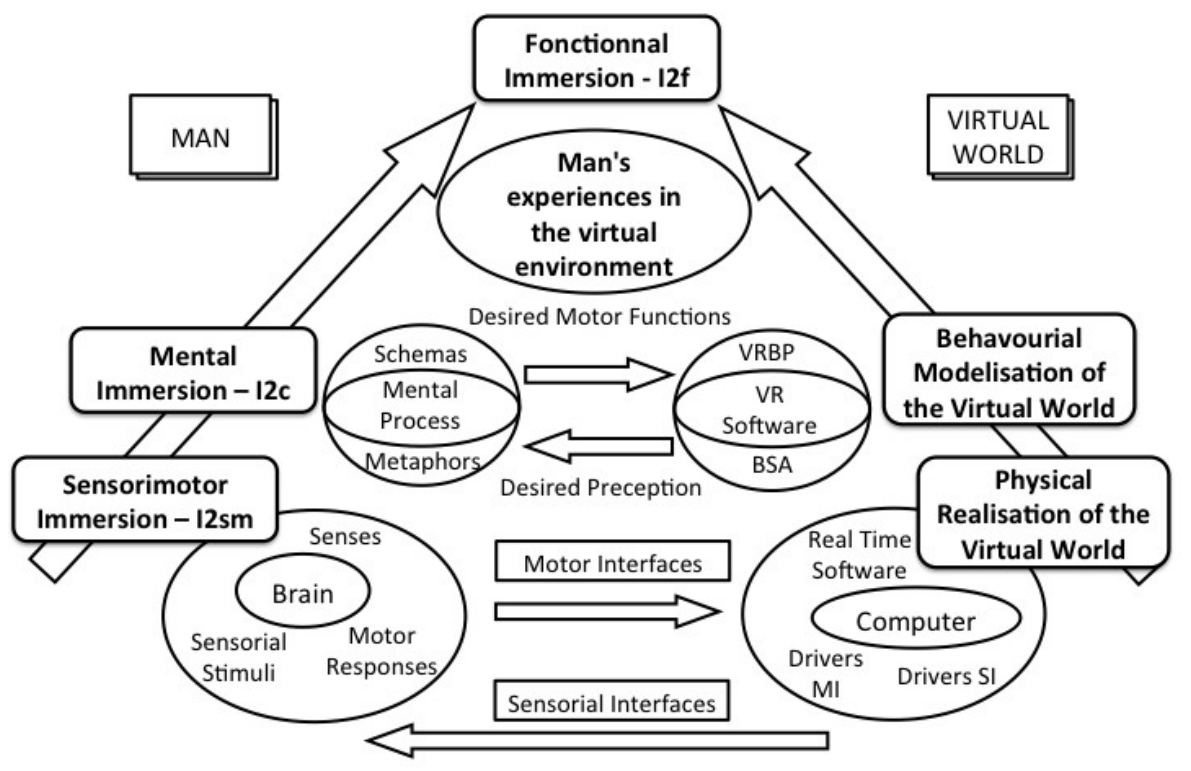

Figure 1. Immersion and interaction of man in a virtual environment [3]. 


\subsection{The various phases of the $I^{2} I$ method}

After having described the theoretical foundations of our method, we are now going to describe its 11 phases in detail (Figure 2). In order to facilitate understanding of the method, we will imagine we are in the following situation: a project manager has just been told by his company to put a team and design a process to create, develop and evaluate a system using virtual reality technologies.

We will also present examples of concrete applications in order to facilitate understanding of our method, in particular a virtual store application aimed at studying consumer behavior.

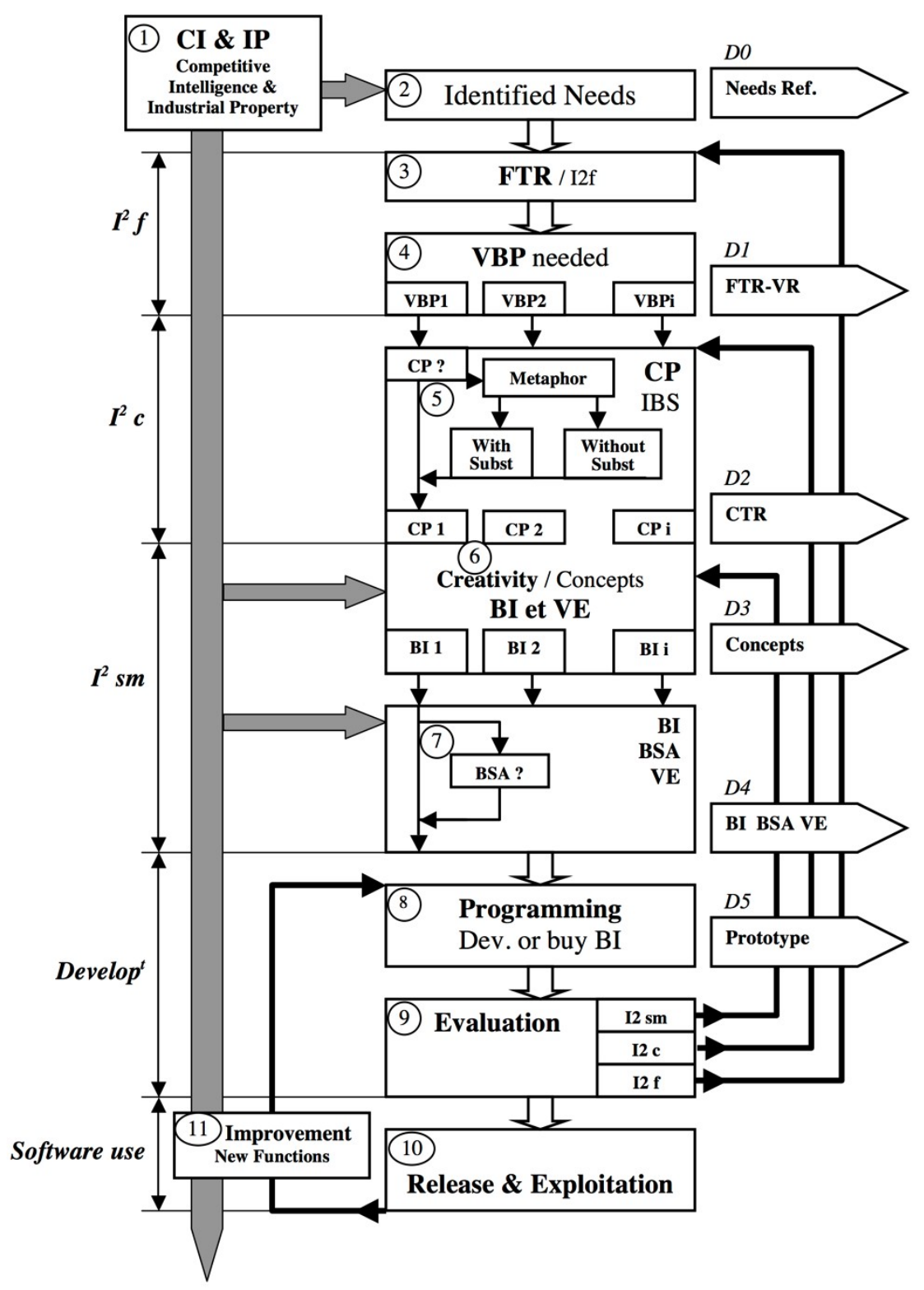

Figure 2. The I²I method - a virtual reality system design method [9] 


\section{PHASE 1: Establishing technological and competitive intelligence and industrial property.}

We can never insist enough on the importance of intelligence while developing systems incorporating new technologies [2]. Setting up technological and competitive intelligence systems can make use of an existing service in the company, which we will ask to monitor a certain number of areas and companies. It can also be subcontracted to an outside organization or entrusted to one or several members of the project team. This initial phase is important and furthermore continues throughout the life of the project. It may also provide an opportunity for the team to update its knowledge before starting the project. The team will therefore discover the existence of systems corresponding to the project goal or may even render the project obsolete.

\section{PHASE 2: Identifying and validating the need to design the system}

Obvious for any designer, this preliminary phase to launching the project is aimed at properly defining the sponsor's needs in relation to developing the system: why is it needed? What is the system supposed to do that is new? What actions, which cannot be done in the real world, does the system make possible? What does the system make it possible to improve? What are the benefits for the company? What are the key factors for success? (= How will the success of the project be judged?) Is the sponsor aware of the cost of developing such a system? What will the return on investment be in relation to a real system? Is there an existing system that is sufficient? Have any other similar systems already been developed? What will be the uses in the future? Do we have access to sufficient human, technical and financial resources? [8]

Virtual reality imposes specific requirements that will need to be satisfied in order to determine whether it will be able to meet the sponsor's needs. In the first instance we should point out that the objective of virtual reality is not necessarily to simulate reality as best possible. It is however an idea that is often generally accepted [5]. The purpose of virtual reality could be to symbolically represent phenomena, concepts or simulate certain aspects of reality. In a very general way we can state that virtual reality capability range make it possible to change time (past or future), place (geographically distant or virtual collaborative space) or type of interaction (symbolic or natural) [6].

This phase ends by constituting the design team around a project leader with all the competencies required to achieve the goals of the project.

\section{PHASE 3: Functional Terms of Reference (FTR) and establishing the functional immersions and interactions (I2f).}

We will not be expanding here on the method for drawing up a FTR. Those interested should refer to the numerous publications and course materials on the subject [12]. The FTR is produced by the design team and finalized by the project leader. It aims to properly formalize ("translate") the design need for the new system and in functional terms clearly define the primary and secondary functions (or "compulsory functions") that the future system should provide. In the framework of developing systems incorporating VR, this phase makes it possible to define the I2f (functional immersions and interactions), as recommended in the "3I2" method [6]. For example, a virtual store must "allow the person to choose a product from among others on a shelf and be able to freely observe him or her" (Figure 3 ). This primary function ("allow...") is detailed and quantified in the FTR and will make it possible to determine the fundamental VBPs and specify the characteristics of the virtual environment (VE).

\section{PHASE 4: Establishing the required VBP (Virtual Behavioral Primitives) and the EV characteristics.}

As we have indicated, the VBP are basic sensorimotor and cognitive activities that will enable the user to realize the functional $\mathrm{I}^{2}$ for which the system is designed. There are 4 main categories of VBP:

- observe the virtual world;

- move around in the virtual world;

- interact in the virtual world;

- communicate with others or with the application. 
The useful VBPs are determined on the basis of the I2f indicated in the FTR. For example, with regard to the virtual store for analyzing buying behavior [3], we determine 3 fundamental VBPs: the observation of the product / three dimensional handling and the spatial orientation of the product / observation of the products on the shelf. We therefore also define 2 secondary VBPs: the ease with which the customer moves and orients in the virtual store (VE) / the possibility of the customer picking up a product and then putting it back on the shelf or in the trolley.

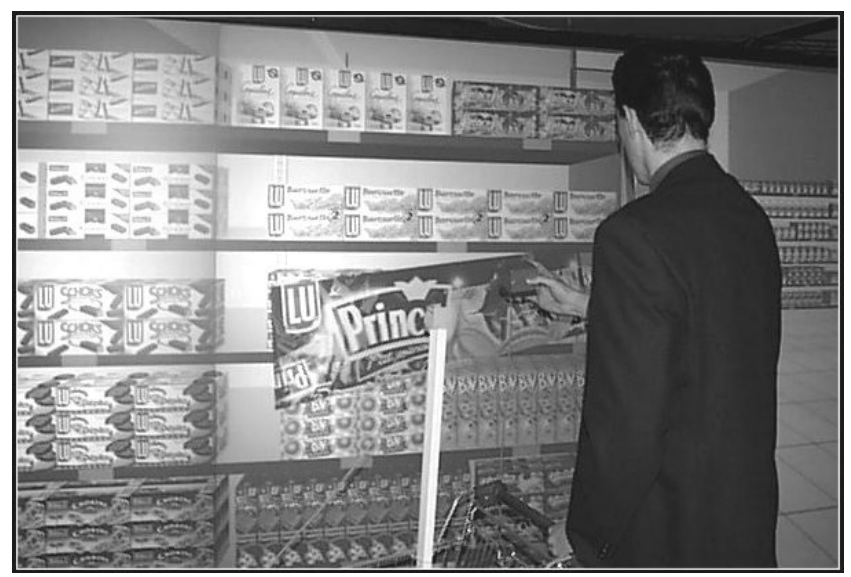

Figure 3. Virtual store for IN VIVO, designed and produced by the Ecole des Mines ParisTech and by the company Sim Team (photo EMP)

Establishing the required VBPs has to be very precise: each VBP has to be analyzed to establish the minimum characteristics that will meet the I2f. For example, if an object has to be handled: should it be handled with two hands or one hand? With 6 degrees of freedom or less? With 360 degree rotation or less? Over what maximum translated distance? Should the weight and inertia be felt? Etc. However, determining the required VBPs and their minimum characteristics should not in any way result in or presuppose technological solutions that will be determined in the next phase.

This phase should also allow the software characteristics to be established in order to create the virtual environment (VE) that meets the needs of the application and therefore the I2f. The VE should be mainly determined at the level of the entities making it up (objects, living beings, fluids, etc.) and at the level of the laws operating there - physical laws (or not) (optical, mechanical, acoustic, etc.) - and the entities' behavioral laws (fixed or variable, deterministic or not, acoustic, etc.). This phase, for the software characteristics needed for the VE is in part related to determining the VBPs, For example in a virtual store, if the user has to have a certain quality in visually observing the products (quantified by visual acuity), the display characteristics of the VE must be linked to the required visual quality.

The question of observation refers back to perception: "what information is needed and will be sufficient for the user to complete the task?" How does the user know that this or that action is required, how does the user know whether the action is successful or not? For example, a maintenance operator on a dangerous site must check that s/he is in fact in nominal mode and that the procedure takes place as it should. S/he should be attentive to the signs that could change the situation into fail mode. It may be an element that is functioning abnormally (post condition not achieved), or an expected or unplanned sound, an abnormally high effort, etc. These questions establish which perceptive elements should be put in place and with what precision.

\section{DELIVERABLE 1: FTR-VR}

Phases 2, 3 and 4 result in writing an initial document, the FTR-VR. It is a "classic" FTR with in addition the definition of the VBPs and the VE characteristics corresponding to the functional I2s. 
PHASE 5 \& 6: Choice of the Cognitive Processes (CP) at the level of the I2c (IBS, metaphors, etc.) and Behavioral Interfaces (BI) at the level of the I2sm.

During this phase, the design team first works on the "cognitive" aspects of the system, while knowing that the exploitable CPs for each VBP are dependent on the BIs that will be associated with the CPs. For each VBP and on the level of the cognitive I2, either in principle or in relation to past experience in virtual reality, we look for the Imported Behavioral Schemas (IBS) that will provide efficient immersion and interaction. If it is impossible to find effective and technically achievable IBSs at an affordable cost, we also have the choice of finding an appropriate metaphor, with or without sensorial or motor substitution, that is compatible with the functional $\mathrm{I}^{2}$. In the example of the virtual store, for the 3 fundamental VBPs, the decision is to make use of the visual observation schema in one fixed direction and the product grasping and spatial manipulation schema. For the 2 secondary VBPs, the decision is to use the movement schema by pushing a trolley and the product grasping and spatial manipulation schema.

The design team has to also work on the sensorimotor $\mathrm{I}^{2}$ on the basis of the desired $\mathrm{CP}$. The goal is to determine the senses and / or the motor responses as well as the artifact in the Behavioral Interface (BI), associated with Cognitive Processes chosen. It is recommended that the chosen artifacts (physical interfaces) are easily used by the target audience resulting in minimal learning time for users (for example no more than $10 \%$ of the time required to use the system). The team also determines what virtual environments will be used by the system. In the example of the virtual store, the following concepts are proposed: using a large projection screen in order to visualize the store on scale 1; using a 6 degree of freedom sensor in a cube for handling the products; using a real trolley to move around the store; realistic simulation of the store shelves and the high definition products so packaging information is readable, etc.

The choice of behavioral interfaces will be a compromise between the requirements of different factors, including:

The objectives of the application: in principle the way of reproducing - or not - a certain number of aspects in the task should not be decided on the basis of technological problems or attractions, but because they are considered useful in order to achieve the objectives of the desired application. Thus, the choice of instant movement of the "mouse click" type in the virtual place the user wants to go is both metaphorical and economic in terms of movement but does not allow the user to learn how to move or orientate him or herself. On the other hand, sometimes certain functionalities should not be reproduced in order to avoid overloading the user with information and allow him or her to concentrate on the task.

The technological and economic limitations: some interfaces cannot technically be produced or are too expensive in relation to the economic context in which we are working and given the uses and objectives of the application.

The usability and the cost related to learning how to use the interface: virtual reality interfaces attempt to allow habitual schemas to be used. Of course, the transposition leads to a loss of "naturalness". Sometimes, using a habitual schema needs a significant amount of time to learn; for example, the use of a treadmill in order to reproduce natural walking [7]. Nevertheless, the cost of learning to use the interface should be considered in relation to the objective sought. If these disadvantages are considered too great, it may be helpful to take a more metaphorical approach.

User experience and profile in other areas: depending on the audience and its experience, some interfaces may be more easily used than others. For example, if the users play video games, a Wiimote could easily be integrated into the system with a low user learning "cost".

Compatibility between the interfaces: if each behavior primitive is taken separately, it is possible to give each one the optimal interface, but there is then a risk of difficulties in compatibility arising. For example, using a treadmill may be incompatible with wearing a head mounted display and difficult in a cave with 6 sides.

\section{DELIVERABLES 2 \& 3: Cognitive Terms of Reference (CTR) \& Concepts (Design dossier)}

Phase 5 results in writing the CTR which complements the FTR with a presentation of the cognitive I2 and the IBS used by the system. During the creative phase, the design team defines all the concepts that seem relevant in producing the system. The choice of concepts that will be selected and implemented are often the strategic responsibility of the project's sponsor. The main principle of the design dossier is therefore presenting an overall summary of all the envisaged concepts, with an evaluation of their advantages and disadvantages, in order to allow the decision makers to make their choice. 


\section{PHASE 7: Definition of the BI, VE and BSA (Behavioral Software Assistance)}

Once the concepts have been defined, the design team now has to exactly define the architecture and the expected characteristics of the future system in order to allow the programmers to produce a prototype.

The metrological characteristics of the physical interfaces have to be defined to be compatible with psychophysical characteristics of the senses and the motor responses, in relation to the desired I2 sensorimotor. The team may decide to use standard interfaces or develop specific interfaces for the system. The technological and competitive intelligence put in place at the start of the project is very useful during this definition phase.

As applicable, Behavioral Software Assistance (BSA) is designed to improve the cognitive I2s (remember that there are still sensorimotor inconsistencies and that the BSAs may partially allow the subject's brain to accept these inconsistencies).

\section{DELIVERABLE 4: VE, BI and BSA}

The definition dossier brings together details of all the elements in the system. It is aimed at the people that will finalize the first system prototype, first and foremost the computer specialists. If artifacts are created (physical interfaces), the computer specialists will be assisted by electronics engineers, mechanics, roboticists, members of the design team or act as subcontractors. System plans, mock ups or descriptive schemas complete the definition dossier.

\section{PHASE 8: Programming (Prototyping)}

A system incorporating VR (virtual reality) relies first and foremost on a computer environment (computer, work station, cluster of computers, etc.) to which all the peripheral devices needed for the system are connected. The prototype phase is therefore principally the responsibility of the computer specialists. Even if some software presents an interface that is easily accessible to non programmers, it is illusory to think that a team without a computer specialist would be able to develop an efficient system. With ongoing contact with the computer specialists, the project leader ensures the system definition dossier is followed and will, if needed, learn to communicate with them (computer specialists sometimes have a vision of the system that is structured around blocks of lines of codes and think more in terms of producing computer functions rather the global function of the system) [10]. Regular validation stages must be planned during this phase in order to match the design team's requirements with the computerization possibilities (which are sometimes overestimated).

\section{DELIVERABLE 5: System prototype}

The prototype should benefit from all the functionalities expected from the system. As in the software industry, each prototype is given a version number (we start with "beta" versions and then go on to versions 1.0, 1.1, etc.). In fact, as it is simple to change the program code, the prototypes are not "stable" and very quickly evolve (as soon as the first inevitable bugs have been corrected). During the first prototype trials exchanging files by Internet allows the computer specialists to very quickly respond to the design team's requests and is one of the advantages of working virtually.

\section{PHASE 9: Evaluation}

It is essential to evaluate a system incorporating VR and it must be done rigorously. It should be done on three levels, testing in the following order and without missing a level: the sensorimotor I2, the cognitive I2 and the functional I2

For the sensorimotor I2 the "metrological" characteristics of the artifacts used are measured (physical interfaces). We then compare them to average human characteristics in order to determine the relative sensorimotor deficit for each sense and for each motor response. We then verify that this deficit is compatible with the desired cognitive $\mathrm{I}^{2}$. The most important metrological characteristics for the interfaces are: precision, sensitivity, the scope of use, the frequency of 
measurement and response time (do not rely on the characteristics given by the interface constructor as these depend on the conditions of use).

For cognitive I2, the evaluation is more difficult to quantify. The time taken to learn how to use the behavioral interfaces is a good indicator. If the user masters the system very rapidly, it means that the chosen CP (Cognitive Process) is effective. Classic ergonomic tests are recommended and preferably should be entrusted to ergonomists.

For functional I2s we refer to the VBPs defined in the FTR-VR. When possible, the evaluated function in the system should be compared with the similar function in the real world. In the example of the virtual store: does the consumer behave similarly in the virtual store and a real store?

Specialists from several disciplines and a representative sample of users (user-designer and end user) will be called on to conclude this phase.

\section{PHASE 10: Release \& Exploitation}

The evaluation phase (Phase 8) loops into the various phases in the method if there is a deficiency in the system in relation to certain expected functions. The present exploitation phase only begins once the system has been "approved" by the sponsor. As it is relatively easy to modify a computer program, systems incorporating VR often benefit from frequent updates and continual development through the lifetime of the system.

\section{PHASE 11: Improvements (Phase 7 loop)}

Day to day operation of the system generally results in users wanting additional functions or new virtual environments (presentation of the product in different locations) above all when designing innovative products. The additional expected functionalities then have to be clearly defined, the costs and the development lead in time evaluated before beginning a new programming phase. A further evaluation phase is then recommended, but is not obligatory if the new functionalities do not fundamentally change the way the system is used.

\section{CONCLUSION}

We are proposing a new method for designing systems using virtual reality (VR) technologies. The result of the experience gained by various research teams, this design method benefits from contributions from current VR research. Our objective is to validate and continuously move forward such multidisciplinary design team methods [11].

Our next goal will be to adapt this method to a co-creation context when final users are involved in the design team to create a new compelling VR experience.

The success of the proposed method is based to a large extent on the efficiency of the project leader who heads the design team. Convinced of the performance of the $I^{2} I$ method, the project leader has to identify any obstacles, define the team's and users' needs, motivate the participants... Assisted by computers and networks, collaborative work in a multidisciplinary team should be encouraged. Even in the virtual field, people are central to the process. 


\section{REFERENCES}

[1] Beckhaus, S., Lindeman, R.W. (2010) Experiential Fidelity: Leveraging the Mind to Improve the VR Experience In Book: "Virtual Realities". Dagstuhl Seminar 2008. Springer.

[2] Corsi P., Christofol H., Richir S., Samier H. (2006). Innovation Engineering - the power of intangible networks, ISTE 2006, 400 p., ISBN 13 : 978-1-905209-55-2.

[3] Fuchs P., Nashahibi F., Lourdeaux D. (1999). A theoretical approach of the design and evaluation of a virtual reality device, VRIC Laval Virtual proceedings, 3-4 Juin 1999, Laval, pp. 11-20.

[4] Fuchs P. (2000). Approche théorique de la réalité virtuelle et son exploitation, Mémoire d'Habilitation à Diriger des Recherches, Ecole des Mines, Paris.

[5] Fuchs P., Moreau G., Papin J.P. (2001). Le Traité de la réalité virtuelle, 540 p., www.ensmp.fr/Fr/Services/PressesENSMP, editor «Les presses de l'Ecole des Mines de Paris », ISBN 2911762-34-7, Paris.

[6] Fuchs P. et al. (2003). Le Traité de la réalité virtuelle, version 2, éditeur « Les presses de l’Ecole des Mines de Paris », Paris.

[7] Lourdeaux D., (2001), Réalité Virtuelle et formation : conception d'environnements virtuels pédagogiques. Thèse de doctorat en Informatique temps réel, Robotique et Automatique, École des Mines de Paris.

[8] Richir Simon, (1994). La conception de produits nouveaux dans l'industrie du jouet, 174 p., Thèse de Doctorat, Génie Industriel, Ensam, 1994, nº4-13.

[9] Richir Simon, (2003). Les technologies de la réalité virtuelle pour la conception de produits, Mémoire d'Habilitation à Diriger des Recherches, Université d'Angers.

[10] Richir S., Fuchs P., (2006a). La méthode I2I, Interaction et Immersion pour l'Innovation. Méthode de conception pour les applications exploitant les environnements virtuels. Editions Techniques de l'ingénieur, Rubrique Réalité Virtuelle, Paris, 20 p.

[11] Richir S., Fuchs P., (2006b). Réalité Virtuelle et Conception, Chapitre du volume 4, les applications de la réalité virtuelle, du traité de la réalité virtuelle, Fuchs P., MOREAU G. et 72 auteurs, 4 volumes, Les Presses de l'Ecole des Mines de Paris, <http://www.esnmp.fr/Presses>www.esnmp.fr/Presses, mars 2006, ISBN 2-91176265-7.

[12] Yannou Bernard, Christofol Hervé, Jolly Daniel, Troussier Nadège, (2008). La conception industrielle de produits, Vol.3 - Ingénierie de l'évaluation et de la décision, série Productique, Hermes Sciences Lavoisier, 321p., ISBN 978-2-7462-1920-4.

\section{GLOSSARY}

BI $=$ Behavioral Interface (IC) ; BSA = Behavioral Software Assistance (ALC) ; IBS = Imported Behavioral Schema (SCI) ; FTR $=$ Functional Terms of Reference $(\mathrm{CDCF}) ; \mathbf{B I}=$ Behavioral Interfaces $(\mathrm{IC}) ; \mathbf{V E}=$ Virtual Environment $; \mathbf{V B P}=$ Virtual Behavioral Primitives $(\mathrm{PCV}) ; \mathbf{C P}=$ Cognitive Processes $(\mathrm{PC}) ; \mathbf{C T R}=$ Cognitive Terms of Reference. 